\title{
Tumores sólidos pseudopapilares en el departamento de patología de la Universidad de Antioquia: serie de casos
}

\section{Solid pseudopapillary tumors in the pathology department of the Universidad de Antioquia: Series of cases}

\author{
Melissa Alejandra Quintero-Picón, ${ }^{*}{ }^{*}$ (1) Germán Osorio-Sandoval. ${ }^{2}$ (1)
}

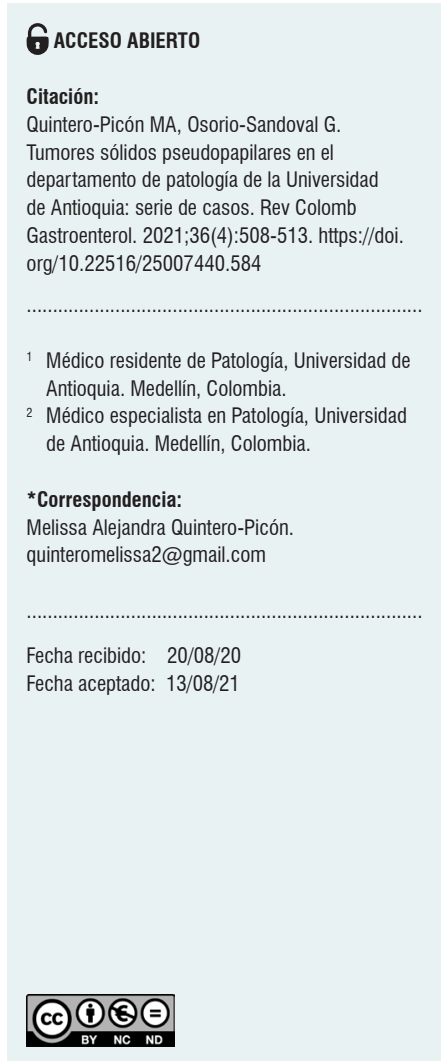

\begin{abstract}
Resumen
Introducción: el tumor sólido pseudopapilar corresponde al 1\%-2 \% de todos los tumores sólidos del páncreas, con un curso poco agresivo. Serie de casos: en el departamento de patología de la Universidad de Antioquia se diagnosticaron 16 casos entre enero de 2004 y julio de 2019. Dos de los casos correspondían a pacientes pediátricos, uno de ellos de sexo masculino con posterior metástasis hepática, dos a mujeres mayores de 40 años y el resto a mujeres entre 17 y 26 años. Un caso presenta células de aspecto sarcomatoide, otros dos presentaron células gigantes multinucleadas y hubo un caso con pleomorfismo grave y presencia de mitosis atípicas. Se observó invasión de la cápsula en 6 de los casos, uno de ellos con invasión linfovascular y 3 casos con invasión perineural. Todos los casos mostraron hemorragia o necrosis, y presentaron en el perfil de inmunohistoquímica positividad para $\beta$-catenina, CD10, receptores de progesterona y CD56. La sinaptofisina fue positiva focalmente.
\end{abstract}

Palabras clave

Tumor sólido pseudopapilar, neoplasia.

\begin{abstract}
Introduction: Solid-pseudopapillary tumors represent $1 \%-2 \%$ of all the pancreatic solid tumors, with low malignant potential. Series of cases: In the department of pathology at the Universidad de Antioquia were diagnosed sixteen cases between January 2004 and July 2019. Two of those cases were pediatric patients, one male with subsequent hepatic metastasis. Two additional cases were represented by two females older than 40 years old and the rest of the cases were females between 17 and 26 years old. One of the cases had sarcomatoid aspect cells, two others revealed multinucleate giant cells, and one last case of severe pleomorphism and presence of atypical mitoses. Capsule invasion was observed in 6 cases: one of the cases with lymphovascular invasion and the other 3 cases with perineural invasion. All cases showed hemorrhage or necrosis, and the immunohistochemical profile was positive for $\beta$-catenin, CD10, progesterone receptors, and CD56. Synaptophysin was focally positive.
\end{abstract}

Keywords

Solid-pseudopapillary tumor, Neoplasia.

\section{INTRODUCCIÓN}

El tumor sólido pseudopapilar, descrito inicialmente por V.K. Frantz en 1959, fue conocido con el epónimo hasta 1996 cuando la Organización Mundial de la Salud (OMS) lo designó como neoplasia sólida pseudopapilar. Es una neoplasia maligna de bajo grado y diferenciación celular incierta, que corresponde al $1 \%-2 \%$ de todos los tumores sólidos del páncreas. Tiene un curso benigno con una supervivencia a los 5 años de $95 \%$, por lo cual se considera 
resuelto con el tratamiento quirúrgico; hacen metástasis en un $10 \%$ a $15 \%$ de los casos, casi exclusivamente a hígado y peritoneo, y se localizan principalmente en el cuerpo y la cola del páncreas. Afecta principalmente a mujeres con un promedio de edad de 30 años ${ }^{(1-4)}$.

\section{SERIE DE CASOS}

En el Departamento de Patología de la Universidad de Antioquia, entre enero de 2004 y julio de 2019 se han diagnosticado 16 casos de tumores sólidos pseudopapilares del páncreas. Dos de los casos correspondían a pacientes pediátricos, uno de ellos de sexo masculino que 7 años después del diagnóstico y cirugía se presentó nuevamente en la institución con un cuadro de dolor abdominal con progresión tumoral en el páncreas y metástasis hepáticas, invasión capsular e invasión perineural en el estudio inicial;

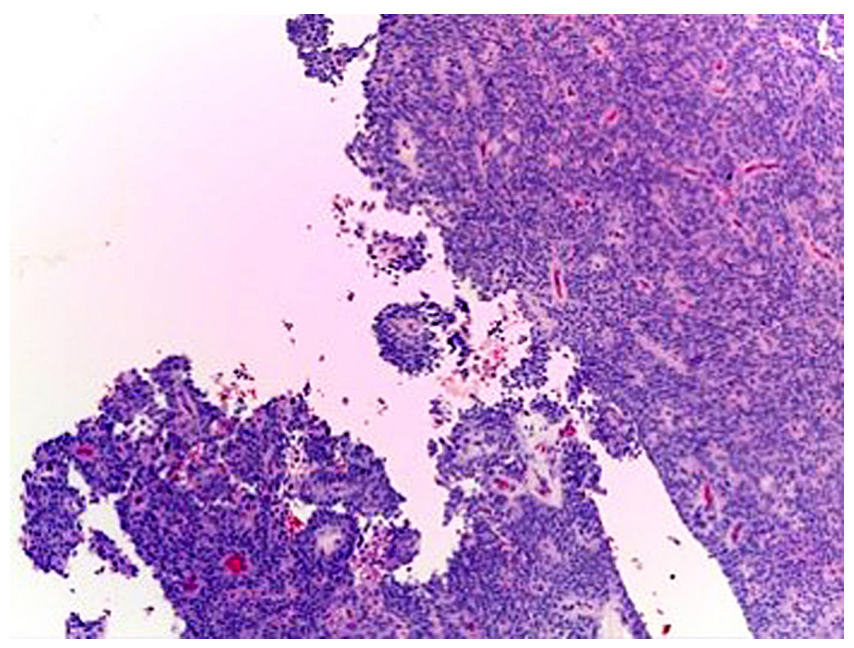

Figura 1. Patrón sólido y pseudopapilar. Aumento: 4X.

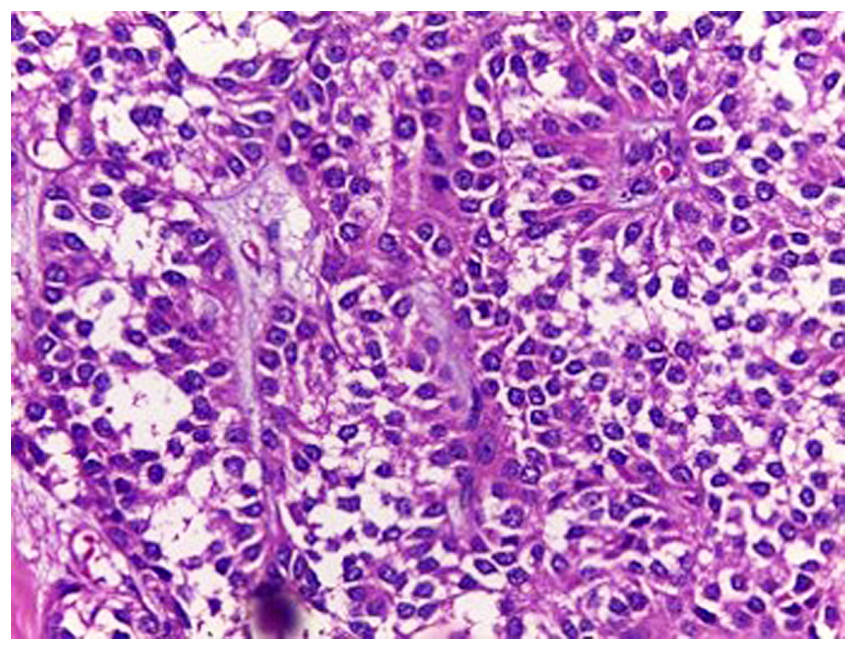

Figura 3. Estructuras pseudopapilares. Aumento: 40X. dos casos correspondieron a mujeres mayores de 40 años, y el resto a mujeres entre 19 y 26 años. Uno de los casos fue diagnosticado en una mujer a la edad de 21 años después de cirugía por trauma abdominal y evolución de más de 20 años con siembras múltiples en el peritoneo.

Histológicamente, todos los casos presentaron un patrón sólido y pseudopapilar, con células de apariencia epitelioide; un caso presentó células de aspecto sarcomatoide y otros dos con presencia de células gigantes multinucleadas. El pleomorfismo nuclear estuvo ausente o fue leve en la mayoría, solo hubo un caso con pleomorfismo grave y presencia de mitosis atípicas, el cual corresponde a una paciente femenina de 56 años. En el resto de tumores, la actividad mitótica fue escasa o nula sin figuras atípicas (Figuras 1-9).

En cuanto a las características morfológicas consideradas como predictoras de comportamiento agresivo, la invasión

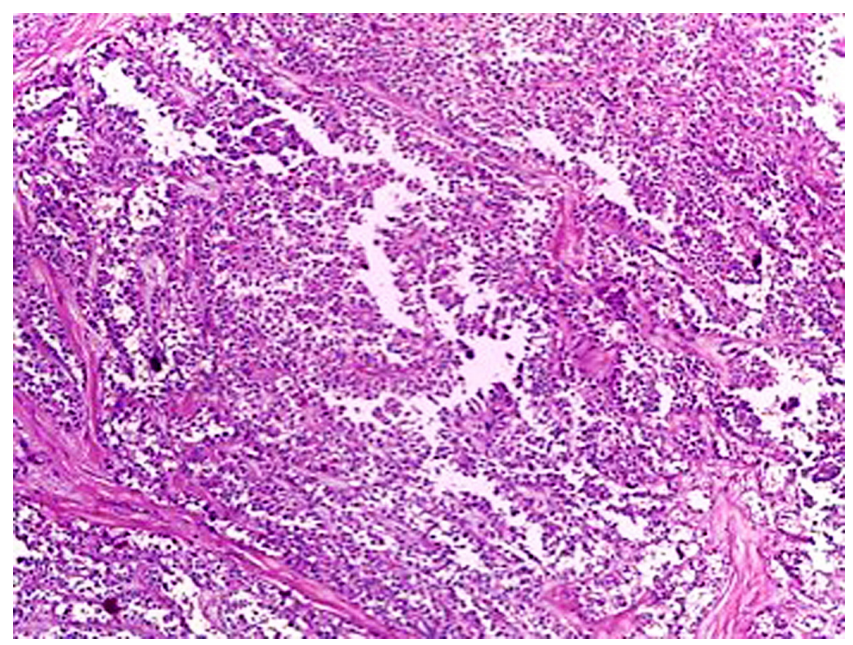

Figura 2. Estructuras pseudopapilares. Aumento: 10X.

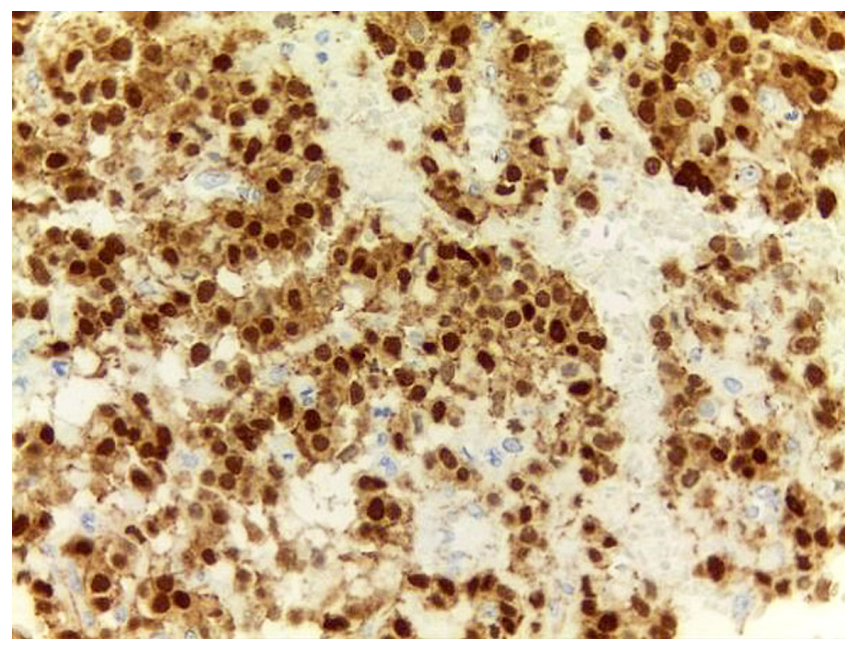

Figura 4. Positividad nuclear y citoplasmática para B-Catenina. Aumento: 40X. 


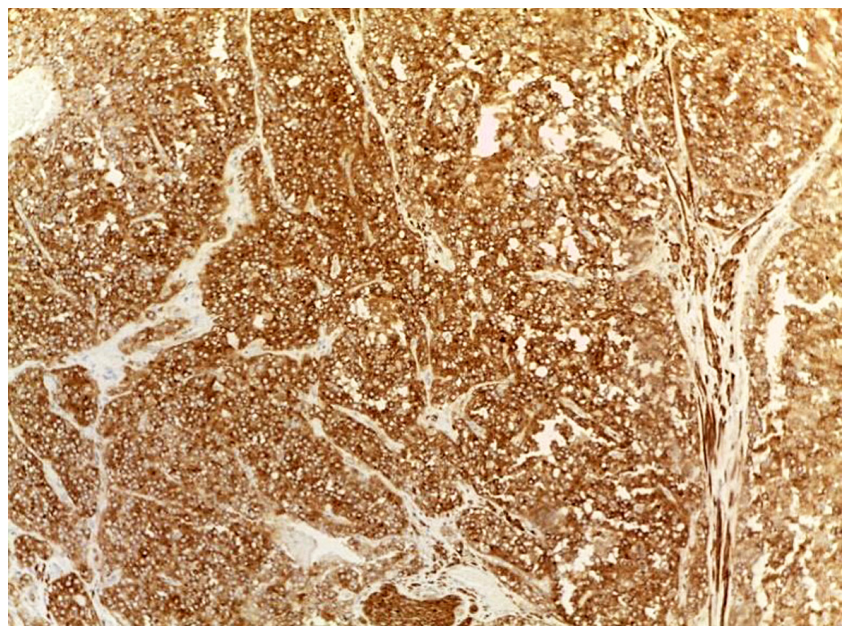

Figura 5. Positividad citoplasmática para CD10. Aumento: 40X.

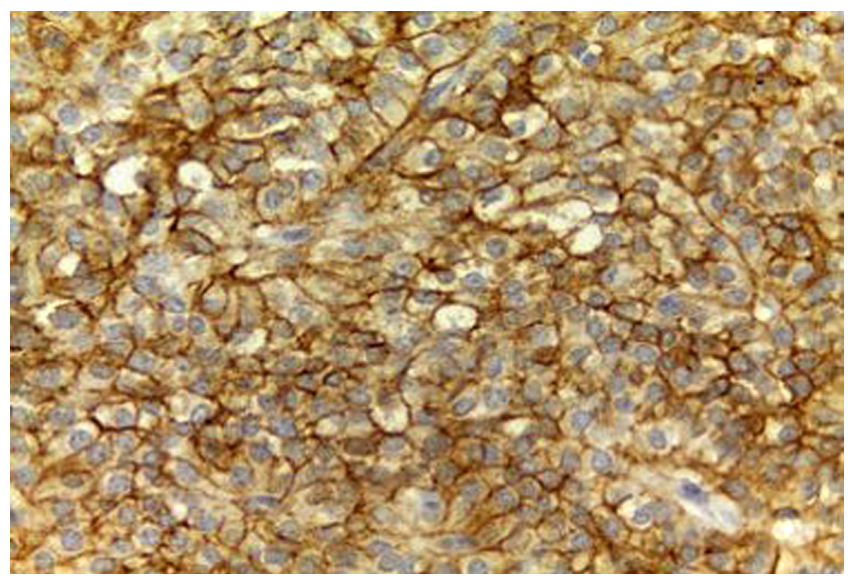

Figura 7. Positividad de la membrana para CD56. Aumento: 40X.

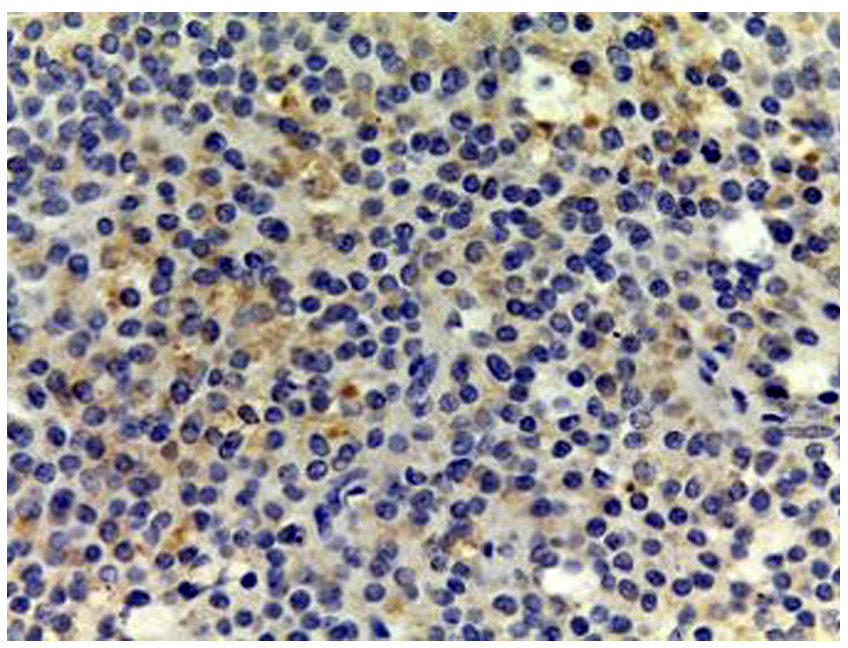

Figura 9. Negatividad para cromogranina. Aumento: 40X.

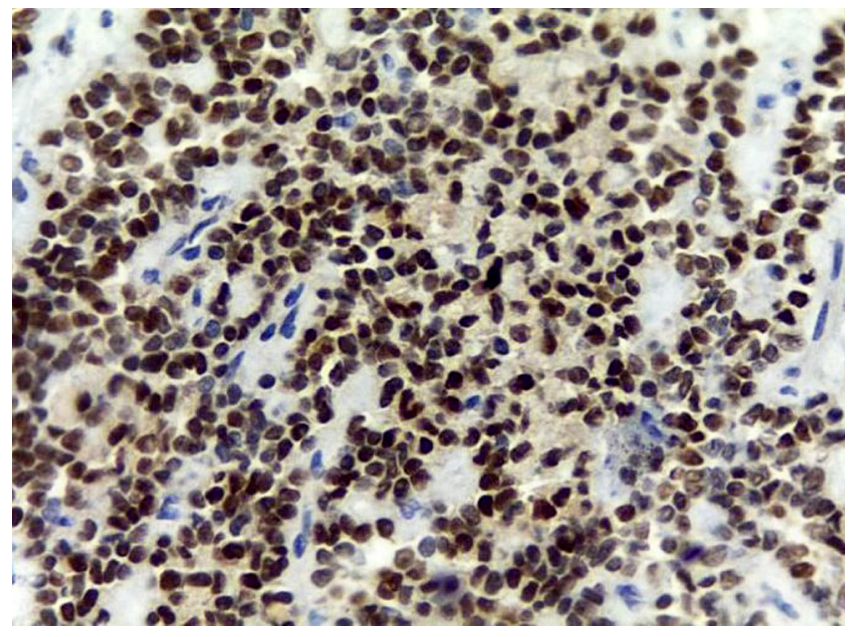

Figura 6. Positividad nuclear para receptores de progesterona. Aumento: $40 \mathrm{X}$.

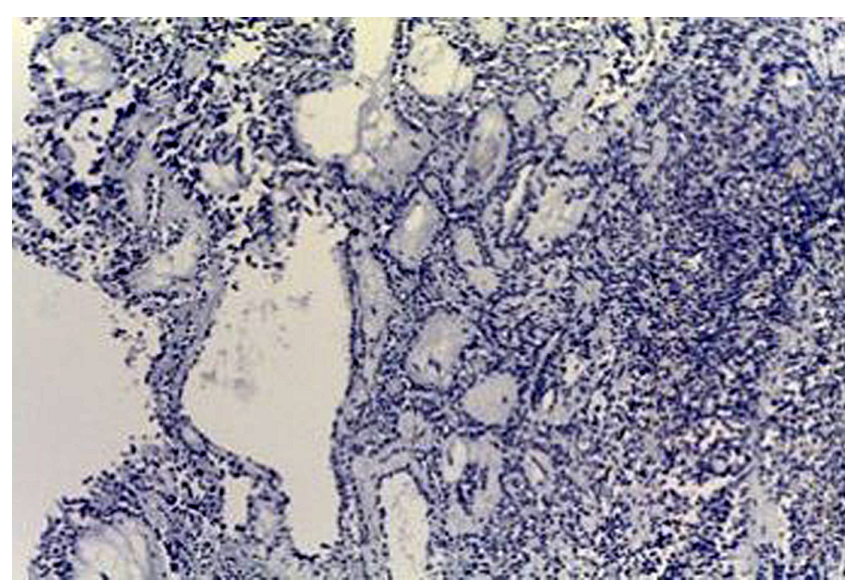

Figura 8. Negatividad para sinaptofisina. Aumento: 10X.

de la cápsula fue la más común, presente en $6(37 \%)$ de los casos; solo uno de ellos presentó adicionalmente invasión linfovascular y perineural en un paciente pediátrico de sexo masculino. Todos los casos presentaron hemorragia o necrosis. En cuanto a los marcadores de inmunohistoquímica, todos los casos (100\%) presentaron positividad nuclear para $\beta$-catenina, otros marcadores positivos fueron los antígenos CD 10 y CD56 en un $43 \%$ del total de casos y los receptores de progesterona fueron positivos en el $100 \%$ de los casos a los que se le realizó dicho marcador, que corresponden al $25 \%$ del total de casos; la sinaptofisina fue positiva en 4 casos, que corresponden al $25 \%$ del total de casos y al $50 \%$ de casos en los que se realizó; la cromogranina y el C-kit fueron negativos en todos los casos. Solo tres casos tuvieron el panel de inmunohistoquímica completo ( $\beta$-catenina, CD10, receptores de progesterona, 
C-kit, sinaptofisina y cromogranina). La positividad de los marcadores previamente expuestos se correlaciona fuertemente con lo descrito en la literatura.

El estudio de inmunohistoquímica se realizó en el laboratorio de inmunohistoquímica del Departamento de Patología de la Universidad de Antioquia, con técnica manual; los casos que no tienen perfil de inmunohistoquímica completo se deben a que en el momento del diagnóstico no se contaba con dicho marcador en la institución (Tablas 1 y 2).

Tabla 1. Características histopatológicas

\begin{tabular}{|c|c|c|c|c|c|c|c|c|c|}
\hline Caso & Arquitectura & $\begin{array}{l}\text { Morfología } \\
\text { celular }\end{array}$ & $\begin{array}{l}\text { Pleomor- } \\
\text { fismo } \\
\text { nuclear }\end{array}$ & $\begin{array}{c}\text { Actividad } \\
\text { mitótica/mitosis } \\
\text { atípicas }\end{array}$ & $\begin{array}{c}\text { Glóbulos } \\
\text { hialinos } \\
\text { intracito- } \\
\text { plasmáticos }\end{array}$ & $\begin{array}{l}\text { Invasión } \\
\text { de la } \\
\text { cápsula }\end{array}$ & $\begin{array}{l}\text { Invasión } \\
\text { perineural }\end{array}$ & $\begin{array}{c}\text { Necrosis/ } \\
\text { hemorragia }\end{array}$ & Otras \\
\hline 1 & $\begin{array}{c}\text { Sólido y } \\
\text { pseudopapilar }\end{array}$ & Epitelioide & Leve & $\begin{array}{c}\text { Escasa/sin } \\
\text { mitosis atípicas }\end{array}$ & Presentes & No & No & Sí/no & Hemosiderófagos \\
\hline 2 & $\begin{array}{c}\text { Sólido y } \\
\text { pseudopapilar }\end{array}$ & Epitelioide & No & No & Ausentes & No & Sí & No/sí & $\begin{array}{c}\text { Esclerosis del } \\
\text { estroma }\end{array}$ \\
\hline 3 & $\begin{array}{c}\text { Sólido y } \\
\text { pseudopapilar }\end{array}$ & Epitelioide & No & $\begin{array}{c}\text { Escasa/sin } \\
\text { mitosis atípicas }\end{array}$ & Ausentes & Sí & Sí & No/no & * \\
\hline 4 & $\begin{array}{c}\text { Sólido y } \\
\text { pseudopapilar }\end{array}$ & Epitelioide & No & No & Ausentes & $\mathrm{N} / \mathrm{A}$ & $\mathrm{N} / \mathrm{A}$ & Sí/no & $\begin{array}{l}\text { Degeneración } \\
\text { mixoide }\end{array}$ \\
\hline 5 & $\begin{array}{c}\text { Sólido y } \\
\text { pseudopapilar }\end{array}$ & Epitelioide & No & $\begin{array}{c}\text { Escasa/sin } \\
\text { mitosis atípicas }\end{array}$ & Presentes & No & No & Sí/sí & Hemosiderófagos \\
\hline 6 & $\begin{array}{c}\text { Sólido y } \\
\text { pseudopapilar }\end{array}$ & Epitelioide & No & $\begin{array}{c}\text { Escasa/sin } \\
\text { mitosis atípicas }\end{array}$ & Presentes & Sí & No & Sí/no & Macrófagos \\
\hline 7 & $\begin{array}{c}\text { Sólido y } \\
\text { pseudopapilar }\end{array}$ & Epitelioide & Leve & No & Ausentes & Sí & No & Sí/sí & Macrófagos \\
\hline 8 & $\begin{array}{c}\text { Sólido y } \\
\text { pseudopapilar }\end{array}$ & $\begin{array}{c}\text { Epitelioide y } \\
\text { células gigantes } \\
\text { multinucleadas }\end{array}$ & Leve & No & Ausentes & No & No & Sí/sí & $\begin{array}{c}\text { Fibrosis, cristales } \\
\text { de colesterol } \\
\text { y macrófagos } \\
\text { espumosos }\end{array}$ \\
\hline 9 & $\begin{array}{c}\text { Sólido y } \\
\text { pseudopapilar }\end{array}$ & $\begin{array}{c}\text { Epitelioide y } \\
\text { células gigantes } \\
\text { multinucleadas }\end{array}$ & Grave & $\begin{array}{c}\text { Escasa/presenta } \\
\text { mitosis atípicas }\end{array}$ & Presentes & Sí & No & Sí/sí & * \\
\hline 10 & $\begin{array}{c}\text { Trabéculas, } \\
\text { nidos y orga- } \\
\text { noide }\end{array}$ & $\begin{array}{l}\text { Epitelioide y } \\
\text { fusiforme }\end{array}$ & Moderado & No & Ausentes & No & No & No/no & Esclerosis \\
\hline 11 & $\begin{array}{c}\text { Sólido y } \\
\text { pseudopapilar }\end{array}$ & Epitelioide & Leve & $\begin{array}{c}\text { Escasa/sin } \\
\text { mitosis atípicas }\end{array}$ & Ausentes & No & No & No/sí & * \\
\hline 12 & $\begin{array}{c}\text { Sólido y } \\
\text { pseudopapilar }\end{array}$ & Epitelioide & No & No & Presentes & No & No & No/no & Hemosiderófagos \\
\hline 13 & $\begin{array}{c}\text { Sólido y } \\
\text { pseudopapilar }\end{array}$ & Epitelioide & Leve & $\begin{array}{l}\text { Escasa/sin } \\
\text { mitosis atípicas }\end{array}$ & Ausentes & No & No & Sí/sí & $\begin{array}{l}\text { Cristales de } \\
\text { colesterol }\end{array}$ \\
\hline 14 & $\begin{array}{l}\text { Pseudopa- } \\
\text { pilar }\end{array}$ & Epitelioide & No & No & Presentes & Sí & No & Sí/sí & $\begin{array}{l}\text { Cristales de } \\
\text { colesterol }\end{array}$ \\
\hline 15 & $\begin{array}{c}\text { Sólido y } \\
\text { pseudopapilar }\end{array}$ & Epitelioide & Leve & No & Ausentes & Sí & No & Sí/sí & $\begin{array}{l}\text { Degeneración } \\
\text { mixoide }\end{array}$ \\
\hline 16 & $\begin{array}{c}\text { Sólido y } \\
\text { pseudopapilar }\end{array}$ & Epitelioide & Leve & No & Ausentes & No & Sí & Sí/sí & $\begin{array}{c}\text { Esclerosis del } \\
\text { estroma }\end{array}$ \\
\hline
\end{tabular}

*No se encontraron otras características. 
Tabla 2. Marcadores de inmunohistoquímica

\begin{tabular}{|c|c|c|c|c|c|c|c|c|c|}
\hline Caso & Vimentina & CD10 & CD117/C-kit & $\mathbf{R P}$ & $\beta$-catenina & Cromogranina & Sinaptofisina & CD56 & Coctel CK \\
\hline 1 & NO & NO & NO & NO & NO & NO & NO & NO & NO \\
\hline 2 & NO & NO & NO & NO & $\begin{array}{c}\text { + nuclear y } \\
\text { citoplasmático }\end{array}$ & * & * & + difuso & * \\
\hline 3 & + difuso & + difuso & NO & NO & $\begin{array}{c}\text { + nuclear y } \\
\text { citoplasmático }\end{array}$ & * & * & + difuso & * \\
\hline 4 & NO & NO & NO & NO & NO & NO & NO & NO & NO \\
\hline 5 & NO & NO & NO & NO & NO & NO & NO & NO & NO \\
\hline 6 & NO & + dot-like & * & NO & $\begin{array}{c}\text { + nuclear y } \\
\text { citoplasmático }\end{array}$ & * & + focal & + fuerte/difuso & NO \\
\hline 7 & NO & + dot-like & NO & + fuerte/difuso & NO & NO & NO & NO & NO \\
\hline 8 & NO & + difuso & * & + fuerte/difuso & $\begin{array}{c}\text { + nuclear y } \\
\text { citoplasmático }\end{array}$ & * & * & NO & + focal \\
\hline 9 & + focal & + focal & * & + fuerte/difuso & $\begin{array}{c}\text { + nuclear y } \\
\text { citoplasmático }\end{array}$ & * & + focal & + difuso & + focal \\
\hline 10 & * & + difuso & * & + fuerte/difuso & + citoplasmático & * & * & + difuso & + focal \\
\hline 11 & NO & + difuso & NO & NO & $\begin{array}{c}\text { + nuclear y } \\
\text { citoplasmático }\end{array}$ & * & + difuso & + difuso & NO \\
\hline 12 & NO & NO & NO & NO & $\begin{array}{c}\text { + nuclear y } \\
\text { citoplasmático }\end{array}$ & * & + focal & + difuso & NO \\
\hline
\end{tabular}

*No se realizaron (no fueron solicitados por el patólogo en el momento del estudio).

\section{DISCUSIÓN}

El tumor sólido pseudopapilar afecta principalmente a mujeres jóvenes, con una relación 9:1, se presenta con síntomas abdominales inespecíficos, como abdomen agudo en caso de ruptura de la lesión por trauma o como hallazgo incidental en las imágenes radiológicas ${ }^{(1,4)}$. En La tomografía axial computarizada (TAC) y resonancia magnética (RM) de abdomen se observa una masa bien encapsulada con densidades o intensidades heterogéneas, lo que refleja degeneración quística y hemorragia dentro del tumor ${ }^{(5)}$. Macroscópicamente, alcanzan medidas de $10 \mathrm{~cm}$ o más; son masas bien circunscritas, sólidas y quísticas; las áreas sólidas tienen una consistencia friable y las áreas quísticas presentan necrosis y hemorragia ${ }^{(5-7)}$. Microscópicamente, tienen una arquitectura sólida y pseudopapilar alrededor de los vasos sanguíneos delgados ${ }^{(8)}$. Las células neoplásicas son discohesivas, de aspecto epitelioide con núcleos redondos y hendiduras, no hay atipia nuclear importante y las mitosis son infrecuentes. Las células tienden a alejarse de los vasos sanguíneos y se degeneran, lo que produce las extensas áreas de necrosis; las células que se mantienen alrededor de los tallos fibrovasculares forman una o múltiples capas que dan el aspecto pseudopapilar característico de la lesión. El estroma puede tener grados variables de hialinización. Pueden existir pequeños grupos de histiocitos epitelioides que acompañan la lesión ${ }^{(1,4-8)}$.

Se han descrito tres variantes histológicas: variante de células claras, variante pleomórfica y variante oncocítica; ninguna de ellas se ha asociado con un peor pronóstico ${ }^{(4,9)}$. Se han descrito características histológicas predictoras de comportamiento agresivo como un patrón de crecimiento difuso con invasión de la cápsula, invasión linfovascular y perineural, necrosis extensa, alta tasa mitótica y presencia de áreas sarcomatoides ${ }^{(1,6,10,11)}$. En la microscopia electrónica se han observado gránulos intracitoplasmáticos electrodensos tipo zimógeno ${ }^{(3)}$. Hasta el momento no se ha definido la célula de origen; algunos autores apuntan a que sea una célula pluripotencial del páncreas, mientras que otros sugieren que sea una célula extrapancreática que se introduce al páncreas durante la organogénesis, ya que se han descrito tumores con características similares en los 
ovarios y testículos $^{(12-14)}$. Su fisiopatología consiste en una pérdida de la capacidad de adhesión celular por mutaciones en el gen CTNND1 en el exón 3 y alteraciones en la vía de señalización Wnt/ $\beta$-catenina ${ }^{(5,7,13)}$.

En cuanto a los marcadores de inmunohistoquímica, son característicamente positivos la $\beta$-catenina y los receptores de progesterona en el $100 \%$ de los casos. Son positivos para CD56 en el $98 \%$, la sinaptofisina puede alcanzar una positividad focal de hasta $62 \%$, mientras que la cromogranina es positiva solo en el $13 \%$ de los casos, el CD10 en el 93 \% de los casos, el C-kit en el $81 \%$ y la ciclina D1 en el $71 \%$, mientras que la E-cadherina es positiva solo en el $13 \%$ de los casos; las citoqueratinas pueden ser positivas en el $30 \%$ al $70 \%$. La inhibina, antígeno carbohidrato 19-9 (CA 19-9), mucina 6 (MUC6), proteína caudal homeobox 2 (CDX2), factor 4 de transcripción de unión a octámeros (OCT4) y el antígeno CD30 son negativos ${ }^{(1,4,8,8,15,16)}$.

Entre los diagnósticos diferenciales se consideran algunos adenocarcinomas, tumores neuroendocrinos, melanoma, PEComa, tumores de la corteza adrenal y tumores de células de la granulosa o de los cordones sexuales ${ }^{(1,2,4)}$.

\section{REFERENCIAS}

1. Notohara K, Nari Y, Fujisawa M. Solid pseudopapillary neoplasm: pathological diagnostic and distinction from other solid cellular tumours of the pancreas. Diagnostic Histopathology. 2008;14(6):266-73. https://doi.org/10.1016/j.mpdhp.2008.04.003

2. Bosman FT, Carneiro F, Hruban RH, Theise ND. WHO Classification of Tumours of the Digestive System. Lyon: IARC; 2010.

3. Odze R, Goldblum J. Surgical pathology of the GI tract, liver, biliary tract and pancreas. 3. ${ }^{a}$ edición. Filadelfia: Elsevier; 2015.

4. Erráez-Jaramillo PJ, Ortiz-Hidalgo C. El diagnóstico histológico e inmunohistoquímico de la neoplasia sólida pseudopapilar de páncreas y su diagnóstico diferencial. Rev Esp Patol. 2019;52(3):178-189. https://doi.org/10.1016/j.patol.2019.03.001

5. Hruban RH, Adsay NV. Molecular classification of neoplasms of the pancreas. Hum Pathol. 2009;40(5):612-23. https://doi.org/10.1016/j.humpath.2009.01.008

6. Jun SY, Hong SM. Nonductal Pancreatic Cancers. Surg Pathol Clin. 2016;9(4):581-593. https://doi.org/10.1016/j.path.2016.05.005

7. Terris B, Cavard C. Diagnosis and molecular aspects of solid-pseudopapillary neoplasms of the pancreas. Semin Diagn Pathol. 2014;31(6):484-490. https://doi.org/10.1053/j.semdp.2014.08.010

8. Hirabayashi K, Kurokawa S, Maruno A, Yamada M, Kawaguchi Y, Nakagohri T, Mine T, Sugiyama T, Tajiri T, Nakamura N. Sex differences in immunohistochemical expression and capillary density in pancreatic solid pseudopapillary neoplasm. Ann Diagn Pathol. 2015;19(2):45-9. https://doi.org/10.1016/j.anndiagpath.2015.02.002

9. Tanino M, Kohsaka S, Kimura T, Tabu K, Nishihara H, Sawa H, Kawami H, Kamada H, Shimizu M, Tanaka S. A case of clear cell variant of solid-pseudopapillary tumor of the pancreas in an adult male patient. Ann Diagn Pathol. 2012;16(2):134-40. https://doi.org/10.1016/j.anndiagpath.2010.11.011
10. Kim SA, Kim MS, Kim MS, Kim SC, Choi J, Yu E, Hong SM. Pleomorphic solid pseudopapillary neoplasm of the pancreas: degenerative change rather than high-grade malignant potential. Hum Pathol. 2014;45(1):166-74. https://doi.org/10.1016/j.humpath.2013.08.016

11. Gomez P, Yorke R, Ayala AG, Ro JY. Solid-pseudopapillary neoplasm of pancreas with long delayed liver metastasis. Ann Diagn Pathol. 2012;16(5):380-4. https://doi.org/10.1016/j.anndiagpath.2011.02.008

12. Syriac S, Kesterson J, Izevbaye I, de Mesy Bentley KL, Lele $S$, Mhawech-Fauceglia P. Clinically aggressive primary solid pseudopapillary tumor of the ovary in a 45-year-old woman. Ann Diagn Pathol. 2012;16(6):498-503. https://doi.org/10.1016/j.anndiagpath.2011.04.007

13. Michalova K, Michal M, Sedivcova M, Kazakov DV, Bacchi C, Antic T, Miesbauerova M, Hes O, Michal M. Solid pseudopapillary neoplasm (SPN) of the testis: Comprehensive mutational analysis of 6 testicular and 8 pancreatic SPNs. Ann Diagn Pathol. 2018;35:42-47. https://doi.org/10.1016/j.anndiagpath.2018.04.003

14. Mengoli MC, Bonetti LR, Intersimone D, Fedeli F, Rossi G. Solid pseudopapillary tumor: a new tumor entity in the testis? Hum Pathol. 2017;62:242-243. https://doi.org/10.1016/j.humpath.2016.08.011

15. Calvani J, Lopez P, Sarnacki S, Molina TJ, Gibault L, Fabre M, Scharfmann R, Capito C, Galmiche L. Solid pseudopapillary neoplasms of the pancreas do not express major pancreatic markers in pediatric patients. Hum Pathol. 2019;83:29-35. https://doi.org/10.1016/j.humpath.2018.08.010

16. Ud Din N, Arshad H, Ahmad Z. Solid pseudopapilllary neoplasm of the pancreas. A clinicopathologic study of 25 cases from Pakistan and review of Literature. Ann Diagn Pathol. 2014;18(6):358-62. https://doi.org/10.1016/j.anndiagpath.2014.10.001 